DAVID MAULÉN DE LOS REYES

ESCUELA DE DISEÑO

UNIVERSIDAD TECNOLÓGICA METROPOLITANA

SANTIAGO, CHILE

DMAULEN@UTEM.CL
Investigación financiada por CONICYT

2008-2009 como tesis de Magíster en Comunicación en el IcSO UAch, bajo la dirección de Gladys Mujica, Mg. Comunicación Organizacional U. Boston. Actualizada el año 2014.

Fecha de recepción: 19/01/2015

Fecha de aceptación: 12/04/2016 Cómo citar: Maulén de los Reyes, D. (2016). Interfaz y heterarquía: diseños de interacción locales para la independencia colaborativa descentralizada. RChD: creación y pensamiento, I(I), 65-78.

DOI: 10.5354/0718-2430.2016.44313

Revista Chilena de Diseño,

RChD: creación y pensamiento

Universidad de Chile

2016, 1(1)

http://rchd.uchile.cl

\section{Interfaz y heterarquía: diseños de interacción locales para la independencia colaborativa descentralizada}

\author{
Interface and Heterarchy: Local Interactions Designs for \\ Decentralized Collaborative Independence
}

Resumen. El concepto de tecnologías de la información interface, se usa indistintamente para referirse a situaciones proyectuales vinculadas al diseño. Habitualmente asociado como derivación del manejo de empresas, especialmente desde los años 80. Desde un ángulo opuesto, la susceptibilidad para la independencia colaborativa descentralizada fue aplicada de manera experimental en el proyecto institucional estatal chileno; desde los años 20 a los primeros años 70, consiguiendo a fines de este período diversos avances en el ámbito de la informática que hoy son revalorizados. En este contexto el diseñador alemán Gui Bonsiepe, proveniente de la experiencia de la HfG-Ulm, coordinó el desaparecido Instituto Tecnológico de la Corporación de Fomento (INTEC CORFO, 1968-1973). Es aquí donde de cierta manera reformula el proto infomático concepto de interfaz, sintetizando otras iniciativas de diseño de interacción necesarias de resignificar que pueden dar cuenta de un vacío para una crítica a la economía política del espacio de lo simbólico en la posible excepcionalidad orgánica que habría significado esta conceptualización a escala nacional. Se hace, por tanto, necesario reconstituir las principales variables y modelos precedentes que delimitaron esta susceptibilidad "heterárquica". Como situación y susceptibilidad específica de enunciación de un concepto fundamental entre tecnologías de la información y comunicación.

Palabras clave: estado desarrollista, economía política del signo, heterarquía, HfG-UIm Sudamérica, interfaz, vernacularidad y funcionalismo.

Abstract. The concept of information technology: interface is used interchangeably to refer to situations related to design projects. Usually associated with referral of company management, especially since the eighties. From an opposite angle, the susceptibility for decentralised collaborative independence was applied experimentally in the Chilean state institutional project; from the twenties to the early seventies. Getting by the end of this period a number of advances in the field of computer science that are now re-valued. In this context the German designer Gui Bonsiepe, from the experience of the HfG-Ulm, coordinated the disappeared Institute of Technology Development Corporation (CORFO INTEC, 1968-1973). In a certain way, he reformulates the proto-informatic concept of interface, synthesising other design interaction initiatives necessary to give a new meaning that can account of a vacuum for a critique of the political economy of the symbolic space in the possible organic exception which would have meant this nationwide conceptualization. It is, therefore, necessary to reconstitute the primary variables and previous models that delimited this "heterarchical" susceptibility. As situation and specific susceptibility of enunciation of a fundamental concept between information and communication technology.

Keywords: developmentalism state, heterarchy, Hfc-UIm South America, interface, the political economy of the sign, vernacularity and functionalism. 
1. Entrevista realizada a Hellmuth Stuven en Valparaíso, en dos partes, durante el año 2007.

2. El tiempo regular para la ejecución de una obra así, en esa época, era cercano a tres años.

\section{El lugar del diseñador y el potencial político de los documentos en el contexto de las economías del conocimiento}

Para las economías basadas en la producción de materias primas sin valor agregado la diferencia entre utilizar la tecnología y generarla se hace aún más profunda, contradiciendo diametralmente los planteamientos respecto a un contexto "postcapitalista" (Drucker, 1993) donde las "economías del conocimiento" habrían colocado en cierta igualdad de condiciones a los diversos actores. A diferencia de la historiografía, el trabajo del diseño siempre se plantea ser proyectivo y prospectivo. En este proceso la utilización de documentos corresponde a la etapa del análisis, y en la medida que estos son considerados directamente a una vinculación aplicada, los transforma en insumos de acción y/o políticos.

Para economías como la de Chile este tipo de consideraciones cobra aún más relevancia cuando de manera inversa a países como Corea, el abandono por una política orgánica de desarrollo tecnológico de acuerdo con las necesidades propias fue drásticamente interrumpido en los años setenta. Es así que de manera doble la administración de la información, y el diseño de interacción, cobran una importancia estratégica, a la vez que permite la revisión retrospectiva de los avances que podrían proyectar su situación específica, a partir de sus mismos poco conocidos referentes que ya enfrentaron desafíos pendientes que definirían mecanismos propios respecto de procesos de subjetivación.

\section{La formulación del concepto de interfaz en el Cono Sur entendida como susceptibilidad de una época}

En el año 1971, el ingeniero informático Hellmuth Stuven, perteneciente a la Corporación de Mejoramiento Urbano (CORMU) dependiente del Ministerio de Vivienda y Urbanismo creado en 1965, es uno de los que recibe el encargo de resolver la iniciación de un ambicioso proyecto de vivienda social (concepto diferenciado del actual: "solución habitacional"), sin precedentes locales. Debían empezar la construcción de 100 mil viviendas en un año, las que además contemplaban cinco tipos de estructuras diferenciadas.

Stuven entonces decide que para poder coordinar estas 100 mil actividades era necesario utilizar un software llamado Pert, que a fines de los años cincuenta había sido creado para el diseño de los misiles Polaris (1958, EE.UU.) complementario al "método de la ruta crítica" (СРM), un tipo de carta Gantt prospectivo que define a futuro las tareas que no pueden sufrir alteraciones, y las que pueden ser objeto de retrasos sin afectar el objetivo.

En la CORMU, Stuven solo contaba con cinco personas contratadas para coordinar todas las obras en el país. Fue así que se resolvió por un método de trabajo que en teoría de la comunicación se llama: Pirámide Invertida. ${ }^{1}$ Esta técnica apareció durante la Guerra de Secesión en Estados Unidos; también conocida como Pirámide Informativa, respondió a las limitaciones del télex; fundando una forma de hacer periodismo.

Gracias al perfeccionamiento de esta técnica que combinaba la pirámide invertida con el software Pert, este ingeniero y su grupo de trabajo enfocaron una nueva estrategia para la exigencia de término del edificio para la III Conferencia de las Naciones Unidas para el Comercio y el Desarrollo (UNCTAD III), que debía estar en pleno funcionamiento en abril del año $1972 .{ }^{2}$

Stuven se reúne con 75 jefes de obra que se capacitan en la traducción de datos en tarjetas perforadas IBM, dos veces al día. Esta forma de transmisión 


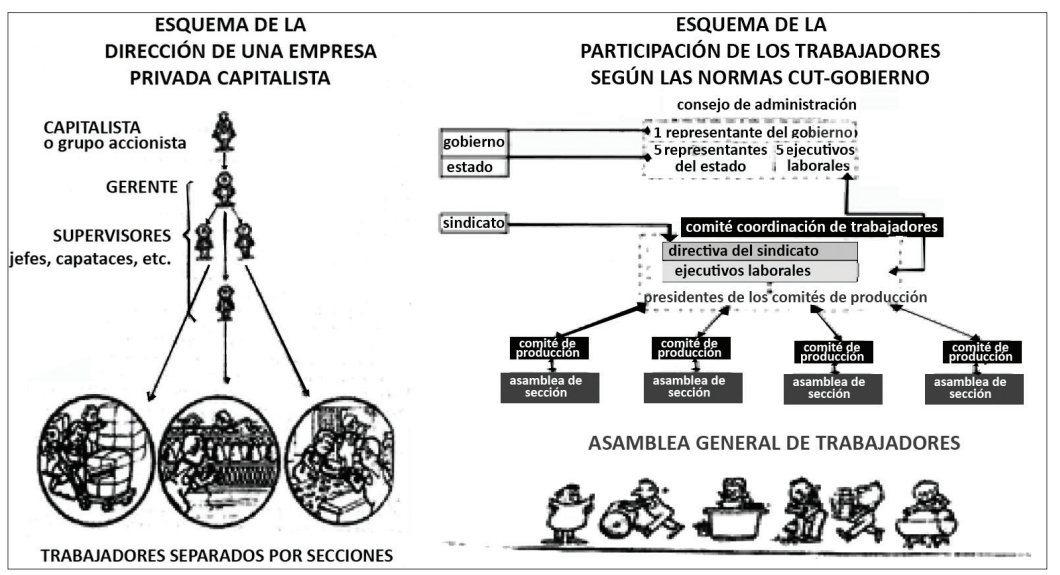

de información fue clave en el éxito del proyecto, pero además se resolvió a través de una susceptibilidad individual y colectiva, para un modo de interacción comunicativo particularmente excepcional.

\section{La heterarquía o independencia colaborativa descentralizada, orgánica y horizontal}

La heterarquía, dividida en agentes y holones, ${ }^{3}$ es un concepto que desarrolla el británico Stafford Beer, para el manejo de la empresa y que en principio significa: lo opuesto a jerarquía (siendo en vez de ser). Este ingeniero investigaba en una época de creciente interés en el impacto que la tecnología podía tener en los procesos socioproductivos.

Es la época de la Escuela de Tavistok, influida por la sicología social del exmiembro de la Escuela de Frankfurt, Kurt Lewin, para entonces referente de la "dinámica de grupo" (Instituto Tecnológico de Massachusetts, MIT). De Tavistok por ejemplo saldrán figuras como R. D. Laing, reconocido autor de una crítica a los condicionamientos sociales de la siquiatría, más conocida como antisiquiatría. A su vez Charles Perrow y Joan Woodward distinguían los tipos de organización mecanicistas, y los orgánicos, más cercanos a la noción de sistema. Beer, proveniente de este período de consolidación del laborismo inglés, tendrá la oportunidad única de implementar sus ideas en la experiencia de transmisión de información en tiempo real, para un sistema productivo bajo condiciones de independencia colaborativa descentraliza horizontales; el Cybersyn o proyecto Synco.

El éxito o fracaso del proyecto Cybersyn dependía directamente de la susceptibilidad sociopolítica en la época, y su realización (real) como sistema de representación sicosocial. Un compromiso colectivo nodal de ser centro y periferia a la vez, operando cada "agente" como microsistema en potencia para todas las funciones que eventualmente pudieran ser requeridas para superar la fragmentación estructural modular. Esa era también la lógica del ingeniero-obrero sintetizada por Stuven. En el caso de Synco el ingeniero informático Isaquino Benadof se basó en el Pert Cpm para crear el software Cyberstride, y en paralelo el rector de la Universidad Técnica del Estado (UTE), el ingeniero eléctrico Enrique Kirberg programaba una red de télex para unir las futuras sedes regionales coordinadas por una sala de computación en Santiago.

Un grupo de estudiantes de Diseño de la Universidad de Chile -Alfonso Gómez, Fernando Schultz, Guillermo Capdevila, Rodrigo Walker-, desarrollaron

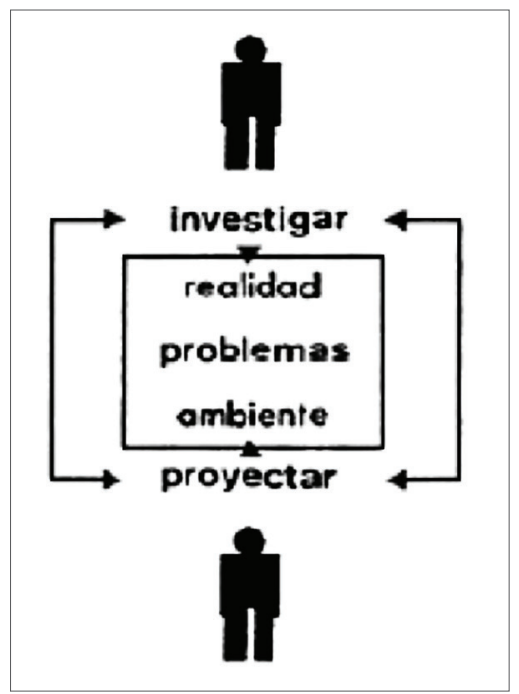

Figura 1. Esquema de Participación Productiva, 1972. Fuente: Harnecker et al. (1972, pp.14-15). Figura 2. Gui Bonsiepe, Investigar-Proyectar, 1972. Fuente: Bonsiepe (1972).

3. Ambas categorías, agentes y holones, corresponden a entidades de operación según la lógica de la independencia colaborativa descentralizada, pero los segundos son menos independientes de las directrices "originales" de un mando central (más horizontales en su forma de organización). 


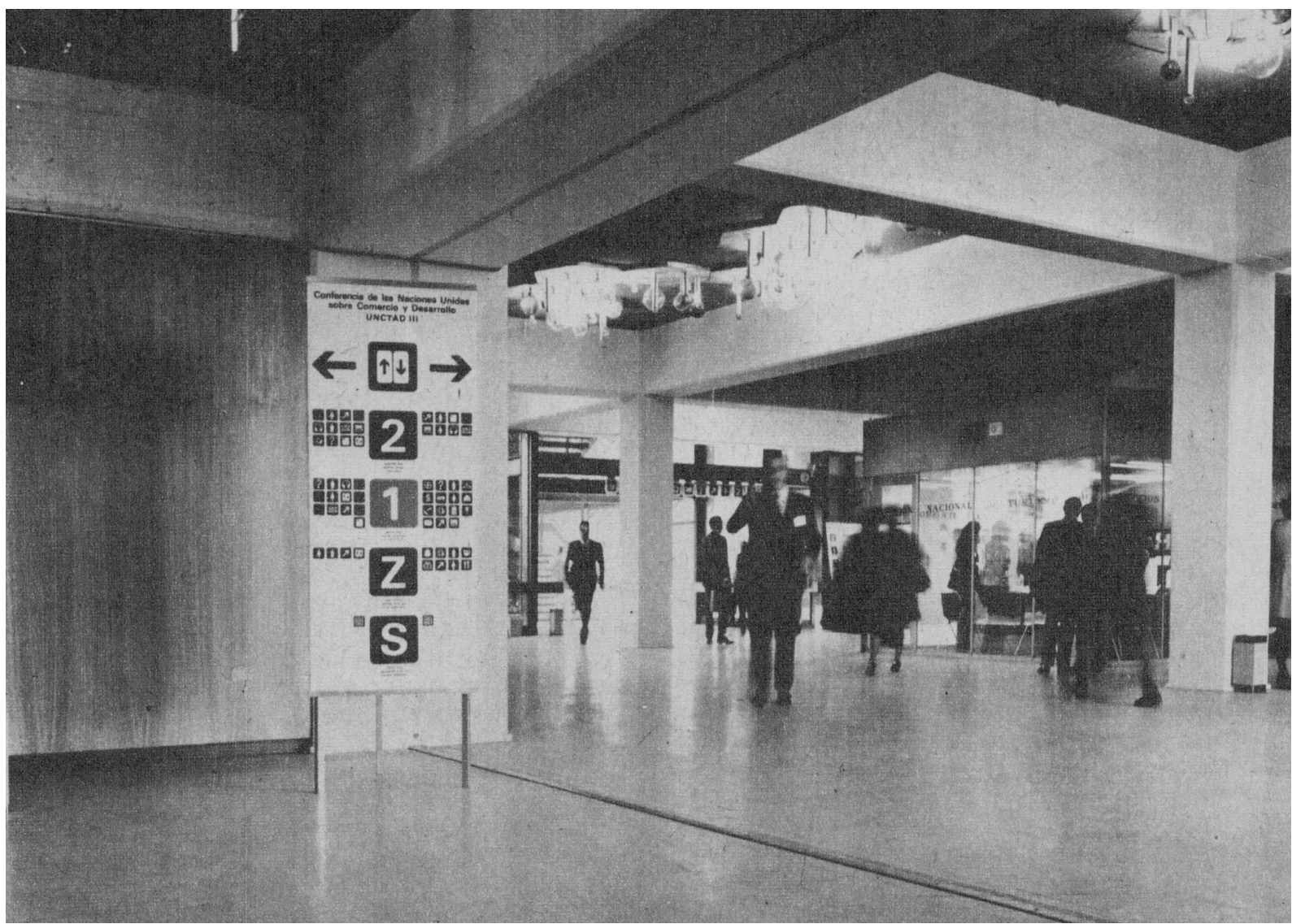

Figura 3. Señalética edificio UNCTAD III, 1972.

Fuente: Cardoso (1972, p.22). las interfaces para el proyecto Cybersyn, y un grupo de estudiantes de diseño de la Universidad Católica -Pepa Foncea, Lucía Wormald, Jessy Cintolesi, Edy Carmona-, desarrollaron el sistema de señalética para el edificio UNCTAD III, ambos con la asesoría del diseñador proveniente de la HfG-Ulm, Gui Bonsiepe, al que luego con apoyo financiero del gobierno alemán se le unirían Werner Zemp y Michael Waiss, también exestudiantes de la HfG-Ulm. Imaginario, Simbólico y Real de la Escuela de Configuración (HfG), ciudad de Ulm, Alemania en una transferencia y reinterpretación diferenciada en dos etapas: a. Incidencias y síntesis enunciativas de Gui Bonsiepe en el INTEC CORFO 1968 - 1973 y la elaboración de un concepto Interfase intentando definir el valor de uso del campo de la estética.

Gui Bonsiepe llega a Chile gracias a la Organización Internacional del Trabajo (OIT) para trabajar en la creación del Instituto Tecnológico de la CORFO (INTEC), como parte de las políticas de sustitución de importaciones (ISI) promovidas entonces por las Naciones Unidas para países que basaban su economía en la explotación de materias primas. Este diseñador venía de participar en una de las experiencias más marcadoras para las "disciplinas" del proyecto desde la Segunda Guerra Mundial. La epistemología desarrollada en la Escuela Superior de Configuración (Hochschule für Gestaltung) de la ciudad de Ulm. La Hfc se consigna como un modelo, retomando los cuestionamientos de los años veinte frente al proyecto de modernidad occidental; es así como fueron construyendo una susceptibilidad y redefiniciones para el legado de la teoría crítica de la Escuela de Frankfurt y la unificación diversa de la ciencia; 


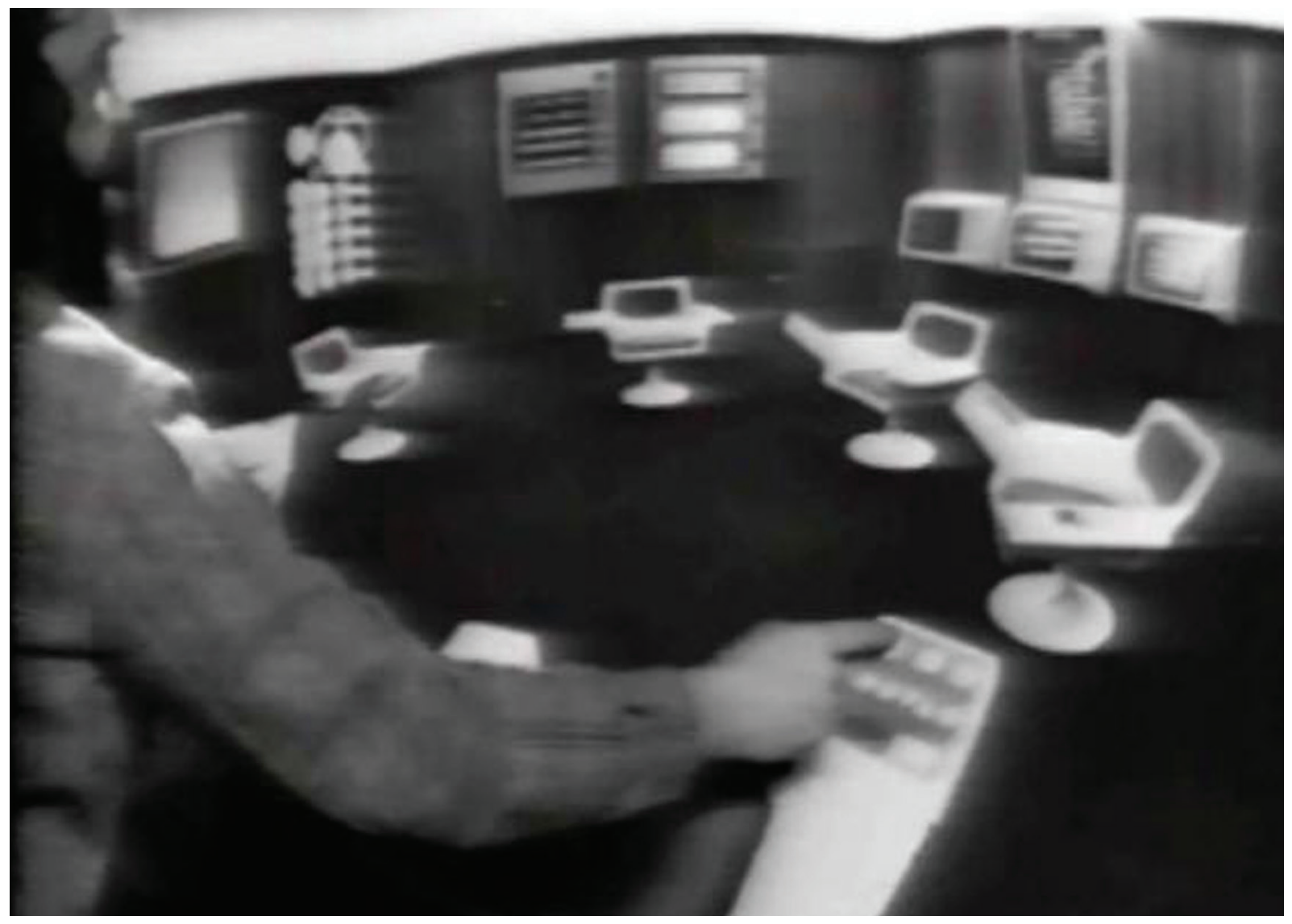

en la experiencia neopositivista de Viena, influidos efectivamente por Ludwig Wittgenstein y las adaptaciones que Max Bense hacía de sus teorías. Este modelo (HfG-Ulm) habría resultado controversial en un país (Alemania) donde el sistema de enseñanza dividido en una segmentada educación patrocinada por el Estado, que además estaba separada en el modelo superior universitario y una más masiva educación técnica profesional.

Como un elemento que connota una idiosincrasia específica, se consiga cómo la educación superior en Alemania respecto de Chile es proporcionalmente inversa, al formarse en ese país, aproximadamente, seis técnicos profesionales por cada titulado universitario.

La HfG-Ulm era distinta a la enseñanza universitaria centrada en conocimiento más bien teórico, pero tampoco basada solo en la enseñanza técnica. Su principal referente era el Instituto de Diseño de Chicago (IIT), institución que había evolucionado desde la "nueva Bauhaus" fundada por un emblemático miembro de la Hfo Bauhaus original -Lázló Moholy Nagy - en esa ciudad. Una equivalencia de la HfG-Ulm con la Bauhaus de los años veinte en Dessau fue la partipación de destacados profesores y estudiantes provenientes de diferentes partes del mundo, incluyendo Sudamérica. Lo cual, en el ambiente posterior a la segunda posguerra marcó una diferencia, porque el cosmopolitismo imperante en la HfG-Ulm venía a terminar a su manera con el aislamiento que habían sufrido los alemanes en relación con los acontecimientos de los últimos años. La elección de un rector suizo - Max Bill- entregaba a la comunidad internacional la garantía
Figura 4. Stafford Beer explicando el Cyberstride en UK, 1974.

Fuente: Fotograma película Cybernetics, part 3, Universidad de Manchester, cortesía Raúl Espejo. Edición digital 2007 Catalina Ossa y Enrique Rivera. 
de que los lazos con la antigua administración pública en manos de los nacional-socialistas no se mantenía, a la vez que conectaba a sus participantes con el debate más actualizado en un sentido global.

Desde otro ángulo complementario, y siguiendo la reposición freudiana que intenta el estructuralista Jacques Lacan, en su época era especialmente significativo reconceptuar la noción signo. Susceptible de ser retrotraída incluso a lo que a fines del siglo diecinueve se alcanza a definir explícitamente como dialéctica (después de la lógica). Deduciendo la "ley de la contradicción excluida" o tercero excluido (principium tertium exclusum). Para una relación A+y A-, es necesario considerar el elemento como situación A. Lacan provocaba con decir: Habitamos en el lenguaje, todo es lenguaje, y más drástico aún; la realidad no existe. Asimilado en su confluencia: la Teoría Crítica de Frankfurt, gracias a Fredric Jameson. Gracias a Frankfurt y Lacan podrá replantear: Cartografía cognitiva, post modernidad, e inconsciente político; radicalizándolos. Antes asociados a un culturalismo positivista, incompatible con este crítico analítico psicosocial.

En la base de estas proyecciones está el aporte semiológico síquico que propone Jacques Lacan, donde diferencia un espacio de significación extrema vinculado a las posibilidades infinitas de significación intraducibles del imaginario, expresando desde otra mirada lo que Roland Barthes llama en cierto sentido poética. Con posterioridad Jameson lo ejemplifica, didácticamente, con la experiencia incompleta y frustrante de narrar un sueño para una posibilidad (o imposibilidad) de estructuración intersubjetiva. El mismo concepto se escapa de las consideraciones hacia el espacio del inconsciente que se deben tener presentes al evocarlo. Conocidas son sus equivalencias entre la falsa consciencia ideológica y el inconsciente político que ensayaran miembros de la tradición de la Escuela de Frankfurt, tales como Herbert Marcuse. A este espacio se contrapone el paso al acto del real psicoanalítico; que se operativiza a través de convenciones simbólicas, una especie de "traducción del fragmento" de todas las interpretaciones posibles que permiten el acuerdo momentáneo (sistemas de referencia = acuerdos de palabra) para pasar de las promesas de real, a un real (mejor dicho realización de) paso al acto. Luego, encima de todo, Jacques Lacan propone que la realidad no existe más allá de estas convenciones particularmente situadas. Si bien existen "demostraciones" más cognitivistas, estas también debieran ser puestas en cuestionamiento según este modelo que se acerca al delicado mecanismo del inconsciente. Fue así que paralelo a las investigaciones de Marcuse, Jean Baudrillard, y los planteamientos que en el mundo empezaban a conocerse como Graphic Use Interfaces (GUI), y antes que Jameson; Bonsiepe siente la necesidad de completar este proyecto de la crítica de la economía política, para poder delimitar un espacio de síntesis e interacción con el que se identificará el contexto de requerimientos de entidades como la CORMU o el proyecto SYNCO, como una susceptibilidad de época que conjuga la articulación de las tres propuestas de conocimiento surgido desde la experiencia sensible (el cómo sé lo que sé de la episteme); según la estructura de Lacan.

Es así que Bonsiepe prepara en 1972 un artículo para la revista INTEC, reeditado para un público más masivo en la revista AUCA (Arquitectura, Urbanismo, Construcción, Arte, palabra que también significa rebelde en lengua mapudungun), que debía salir publicada en su primera parte, durante el mes de septiembre del año 1973, y logró salir un poco tiempo después. Luego la segunda entrega aparecería en el número subsiguiente el año 1974: VI / VI / SECCIÓN del diseño industrial. 


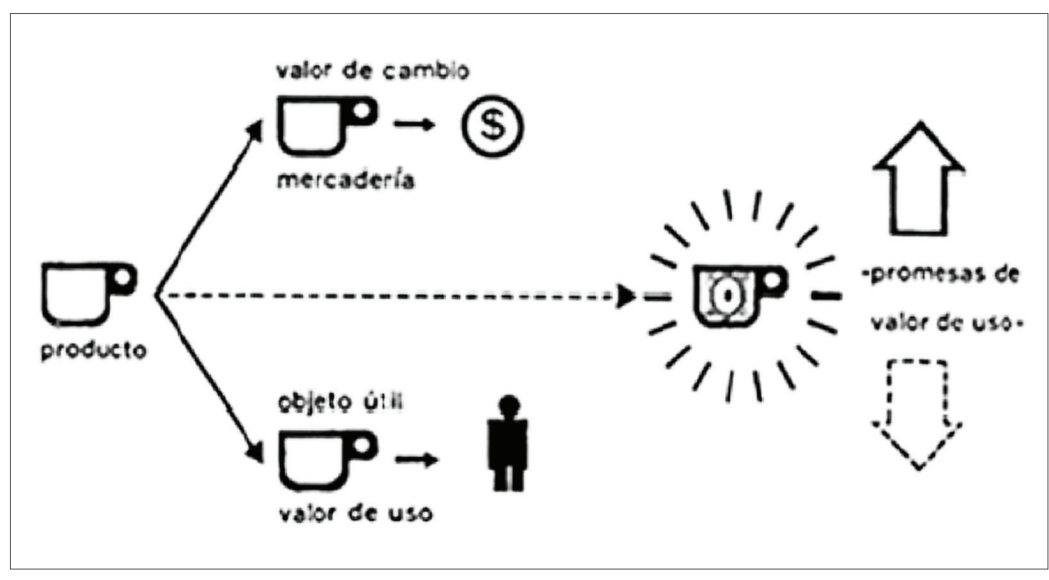

Es en este artículo donde delimita la emergencia del concepto interfaz como consecuencia de intentar calcular el concepto "valor de uso", proveniente de la "economía política", aplicado al campo de la estética como campo subjetivo pero concreto de los procesos de percepción:

En realidad, hay pocas cosas más vagas que las necesidades estéticas, lo que el ritualismo científico casi prohíbe mencionar. Pero por vagas que sean, no por eso dejan de existir. En consecuencia, consideramos la calidad estética o formal de un producto no como un anexo, el cual fácilmente se puede tildar de superfluo o agregar a un producto ex post, sino como factor esencial de su calidad global. Valores puros de uso existen solamente en tal abstracción. Ellos no sobrevivirían un purgatorio contra los supuestos pecados de la estética, pues ella forma el interfaz indispensable para poder realizar (término remarcado en el artículo original de 1973) los valores de uso.

Este análisis y formulación también consideraba otras exigencias de contexto, lo que queda en evidencia en el testimono de Rodrigo Walker al explicitar que Bonsiepe les exige previamente estudiar economía con Pedro Vuskovic; pero el punto principal aquí es destacar las determinantes de enunciación para un concepto estratégico en el diseño de modelos de interacción complejos como eran las áreas de producción de la coRFo bajo los conceptos de colaboración descentralizada independiente (ICD) o "heterarquía" que proponía la lógica del proyecto sYNCO $u$ otros proyectos que se planteaban en ese momento en la educación y otros campos.

b. Colaboración directa de Max Bill en el diseño de un centro comunitario para las cooperativas de villas de autoconstrucción de Eduardo Vargas, con colaboración de Cornelia Koch en Valparaíso, 1960-1966.

Durante el primer período de la HfG-Ulm la dirección estuvo a cargo del diseñador, artista y arquitecto Max Bill, quien también es autor del edificio donde se albergó esta institución. La versatilidad y compromiso de Bill también lo depositó incluso en su posterior trabajo como parlamentario. El plan de estudios de este primer período estuvo caracterizado por una readecuación de los objetivos de su antecedente, la "Bauhaus de Chicago", el IIT fundado por Moholy Nagy, y continuado por Serge Chermayeff, en relación a reunificar las

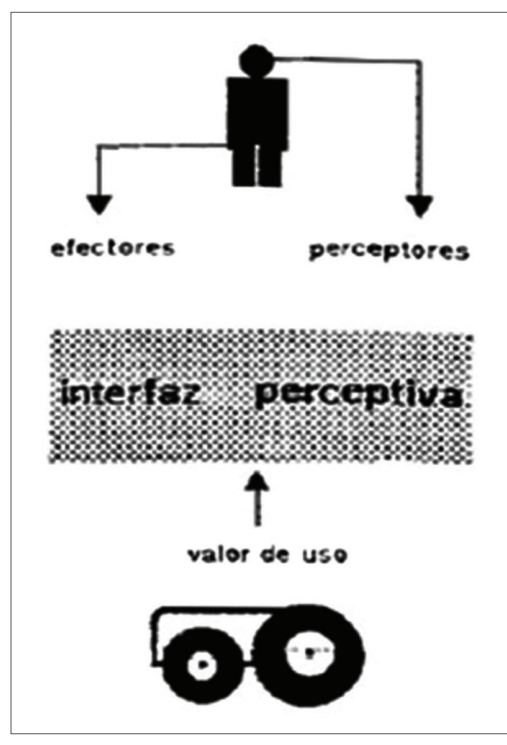

Figura 5. Gui Bonsiepe, Promesa de Valor de Uso, 1972.

Fuente: Bonsiepe (1972).

Figura 6. Gui Bonsiepe, Interfaz Perceptiva, 1972. Fuente: Bonsiepe (1972). 
Figura 7. Eduardo Vargas, Coop Design, Valparaíso 1966.

Fuente: Archivo de Sofía Vargas-Koch.
1960-1966

11.000 habitantes

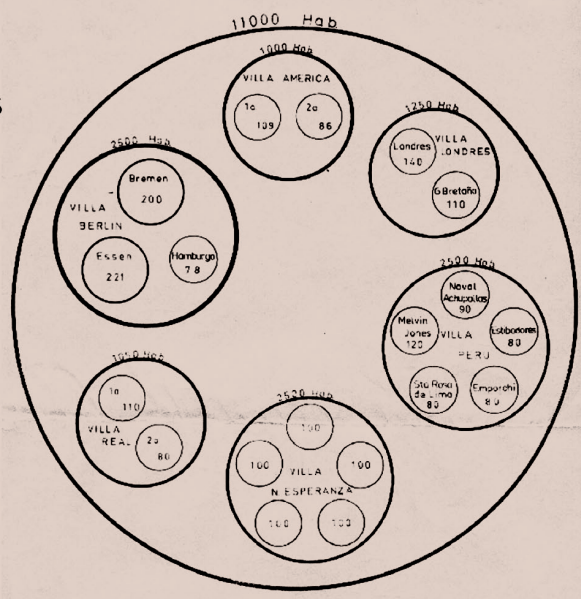

- Viviendas cooperativas, autoconstrucción (800 unidades, $56.000 \mathrm{~m} 2$ ) - 1960-64

- Construcción de viviendas cooperativas (1200 unidades, $84.000 \mathrm{~m} 2$ )

formas de construcción del ser humano fragmentado durante la concepción de modernidad industrial heredada del siglo XIX.

El primer plan de estudios de la Hfo-Ulm volvía a plantear un "curso preliminar" (Vorkurs) que unificaba en los futuros diseñadores conocimientos sicológicos, fisiológicos y analíticos a través de la técnica, de manera que la materialización de cada disciplina fuera evolucionando en una concepción integral de colaboración entre otras disciplinas, para no disociarse del medio ambiente social y natural. De alguna forma estos objetivos recordaban los planteamientos de Walter Gropius sobre la "arquitectura integral" o "total". Con la dimisión de Bill a fines de los años cincuenta también fue suprimido el "curso preliminar", sustituido por asignaturas de introducción a cada especialidad en las cuales desde primer año los estudiantes concurrían a estudiar por separado, y la sección de arquitectura, transformada en construcción industrial, quedó separada de comunicación visual e información, aunque hasta entonces habían tenido cursos en común marcados también por el espíritu original del proyecto respecto de una integración cultural y la necesidad de reflexionar en torno al rol de los profesionales en la sociedad. Desde esta perspectiva el concepto de funcionalidad no era visto de manera aislada sino más bien se planteaba desde consideraciones integrales.

Fue durante el último año en que se impartió esta formación preliminar al conjunto de estudiantes que ingresó el arquitecto Eduardo Vargas, formado en la Escuela de Arquitectura de la Universidad Católica de Valparaíso, Chile. Uno de los elementos fundamentales para que Vargas decidiera estudiar con Bill fue su presentación en São Paulo el año 1953, la cual terminó enfatizando tres directrices básicas para la arquitectura:

1. Debe ser ante todo modesta y clara.

2. Es un arte cuando todos sus elementos (la función, la construcción y la forma) están en perfecta armonía.

3. Es un arte social, y como tal debe estar al servicio del hombre. 
Esta determinación de Bill por una arquitectura social, que fuera más allá del formalismo, fue fundamental en la decisión de Vargas para seguir estudios en Alemania con él. Con el alejamiento de Bill de la institución un conjunto de estudiantes continuó en contacto con él, entre ellos Eduardo Vargas y Cornelia Koch. Ambos trabajaron un tiempo en el estudio de Bill y luego en 1960 viajaron a Chile, donde dirigirían un masivo proyecto de villas de vivienda de autoconstrucción en cerros de la ciudad de Valparaíso.

La formación integral del primer período de la HfG-Ulm también caracterizó un modo de trabajar colectivo que recuerda el Collaborative Architects fundado por Gropius en Estados Unidos, y antes de eso a los movimientos "Coop" en los que participó el segundo director de la Bauhaus, Hannes Meyer.

Durante la ejecución de este proyecto en Valparaíso ambos autores tomaron contacto con Bill, quien fortaleció la idea de organizarlo en cooperativas, metodología que se utilizaba exitosamente en distintas situaciones, sobre todo en Suiza. A esto se suma el interés manifiesto de Eduardo Vargas por los planteamientos de Max Bill antes de ir a Alemania, en relación a rechazar la aplicación formalista y descontextualizada de las ideas de la vanguardia moderna, y en cambio reintepretar las variables que en cada situación específica hacen más viable el proyecto. Dentro de los planteamientos de la arquitectura moderna, entendiendo el concepto de arquitectura en el sentido amplio (diseño o proyección de sistemas o estructuras) cobra relevancia estratégica el diseño de interacción.

Desde esta relación se desprende un concepto articulador, el nodo, que consigue una de sus expresiones más representativas en el planteamiento de Unidades Vecinales, el cual consolida sus principios en un elemento en particular, el diseño de un centro comunitario.

En la planificación urbana de los años cincuenta se alcanzó cierto consenso que se vio reflejado en la planificación orgánica y las consideraciones al diseñar la ciudad como organismo vivo interconectado. Así las relaciones que actualmente se hacen respecto de las redes de colaboración a través de internet tienen diversos antecedentes en la proyectación analógica que se hacía en el urbanismo de esta época.

Una de estas concepciones es la diferenciación entre el sistema modular como principio, contra un horizonte nodal donde cada unidad del sistema contuviese en potencia todas las características del sistema para lograr funcionar así de manera heterárquica, como llegó a plantearlo también la cibernética de segunda generación cerca de 1970.

Esta forma también se traducía en la pedagogía "Coop" de Hannes Meyer y era explícita en los talleres de la Hfc-Ulm de la primera época.

En términos de planificación urbana la Unidad Vecinal funciona como una zona residencial dotada de servicios y equipamiento que le garantizan la autonomía y a la vez colabora con el funcionamiento de otras unidades vecinales y con la trama urbana en general. Desde que fueron planteadas, dentro de su programa uno de los elementos fundamentales para consolidar continuamente la cohesión participativa de sus integrantes era el diseño de un centro comunitario gestionado por su comunidad.

Eduardo Vargas nunca perdió el contacto con Max Bill durante el desarrollo del proyecto de villas de autoconstrucción y logró su colaboración directa en el diseño de un centro comunitario con características específicas. Gracias a un financiamiento del servicio de cooperación alemán DAAD, Vargas se reunió con Bill en su taller y proyectaron el respectivo centro comunitario, interpretando algo que a Vargas le llamaba especialmente la atención 
en Bill: su interés por observar las características funcionales desarrolladas por la arquitectura vernacular, en este caso la casa colonial con patio central, en vez de una aplicación mecánica de una tipología moderna que en realidad en cada lugar había dado solución a problemas particulares del ambiente donde sería emplazada. Con posterioridad a este proyecto esta observación también se distingue en otro representante del movimiento moderno chileno, Emilio Duhart, en su decisión para el edificio de la Comisión Económica para América Latina CEPAL, y en el Aeropuerto Internacional de Santiago de Chile. Solución que en su funcionalidad pasa inadvertida para la mayoría, que solo la interpreta como arquitectura moderna. Esta forma de pensar asume desafíos complejos, pero que proyectan el diseño a soluciones con mayor éxito, pero además pone en entredicho la concepción verticalista de las soluciones, y aquí se desprende un principio aún más fundamental en el éxito de esta experiencia con raíces en los postulados de la primera fase de la HfcUlm: el concepto de autoconstrucción, claramente una reinterpretación de la teoría del nodo y de la heterarquía (independencia colaborativa descentralizada) que se materializa en una forma específica de diseño de interacción. La cohesión social como programa arquitectónico es un objetivo principal en el diseño de los centros comunitarios, construye ciudadanía, pero antes de eso, la metodología de trabajo a través de las cooperativas de autoconstrucción significó en la época un cambio de paradigma, como lo sería después la capacitación que hace Stuven a los jefes de obra para que ingresen la información en tarjetas IBM durante la construcción del edificio para la UNCTAD III, en vez de ser exclusivamente el ingeniero calificado el que realice esa función. Quebrando esa lógica el éxito del proyecto de Cornelia Koch y Eduardo Vargas, con la colaboración directa de Max Bill, no hubiese sido posible sin el grado de cohesión que se dio durante todo el desarrollo del proyecto en cada uno de los participantes del modelo de autoconstrucción. Esta concepción de diseño de interacción se dio en otros proyectos de la época, como la población La Victoria, o la posterior Villa La Reina, pero esta vez con la excepcional aplicación y reintepretación de los principios de la HfG-Ulm.

En parte esta reintepretación se ve en la influencia del currículo de la primera época, donde las asignaturas dedicadas a materias políticas y sociales interrogaban continuamente a los estudiantes por su responsabilidad como profesionales en el mundo que les habría tocado ejercer. A su vez, en un sentido ampliado del diseño de interacción, este proyecto se distinguió por cuanto sus responsables se fueron a vivir al lugar de construcción, estando a la par de los otros moradores que día a día levantaban las obras. Dentro de la línea de participación se puede especificar tres niveles: el de la construcción en cooperativas, en este caso con el apoyo de Invica (una constructora de Iglesia), pero también con el sistema de ahorro en un Banco de Cooperativas, y posteriormente con la administración comunal.

\section{5/1945/ 1973: período de constantes avances y retrocesos del modelamiento representativo de interacción, según la línea de un sindicalismo funcionalista orgánico; dinámico, y vernacular}

El planteamiento de Bonsiepe respecto de la mediación o mediatización no es exclusivo de su época de enunciación; más bien debe ser interpretado como la sucesiva articulación de sistemas de referencia que apuntaban de manera crítica a la independencia colaborativa descentralizada, desde un ángulo 


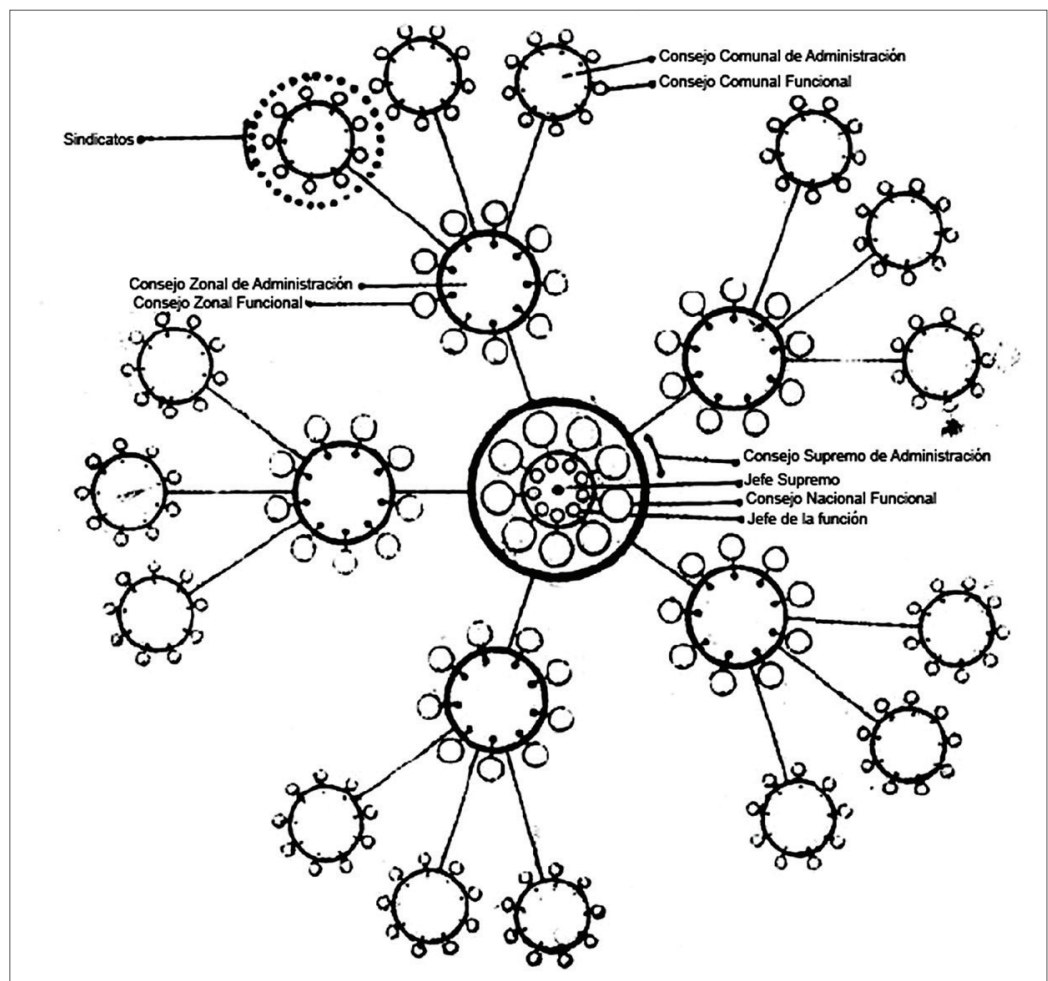

orgánico y horizontal. Las condiciones del modelo Estado-desarrollo intentado por Chile entre los años veinte a los años sesenta, a pesar de los matices, entrega suficientes contrastes (y resistencias) para ejemplificar patrones en la construcción de este espacio convenido como significado y significante. Sin embargo, es necesario también consignar algunos de sus avances y antecedentes más incidentes, tanto en su compleja y ambiciosa estructura de variables, como en sus grados de constitución, superando el campo de producción simbólica de sentido a ser realmente lo que el sociólogo Pierre Bourdieu llama sistema de hechos. Más vinculado con la definición de institución de referencia, pero en este caso reforzando las implicancias que tendría como referencia sicosocial.

El teórico estructuralista-constructivista Jacques Aumont introdujo el concepto dispositivo como conceptualización de los factores que se constituyen en la formulación de una interfaz, hasta donde Bonsiepe la mencionó inicialmente:
Figura 8. Estado sindicalista funcional. Socialista, democrático, unitario, descentralizado, aburocrático, sin clases ni partidos, orgánico. Individuo y Estado se identifican en los grupos funcionales. Fuente: Frente Funcional Sindicalista (1935), p.27.

Las determinaciones fisiológicas y psicológicas de la relación del espectador con la imagen no bastan para describir completamente esta relación. Esta se encuentra además, en un conjunto de determinaciones que engloban e influyen en toda relación individual con las imágenes. Entre estas determinaciones sociales figuran, en especial, los medios y las técnicas de producción de las imágenes, su modo de circulación y, eventualmente, de reproducción, los lugares en que ellas son accesibles, los soportes que sirven para difundirlas. El conjunto de estos datos, materiales y organizacionales, es lo que entendemos por dispositivo. 

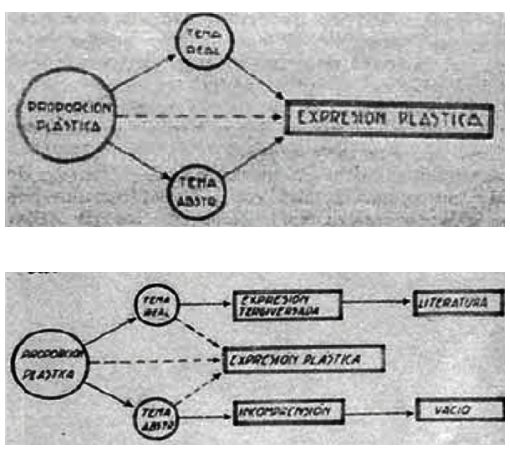

Figura 9 y 10. Waldo Parraguez, Expresión plástica-proporción plástica, 1935. Fuente: Parraguez (1935, pp.11-12).
Sobre esta relación, y la aproximación al concepto de interfase que hace Bonsiepe desde una formulación de una crítica a la economía política del espacio de lo simbólico, resulta aún más interesante la relación etimológica que consigna Aumont para el término imagen:

Notemos de paso que la palabra economía viene de raíces griegas que significan regulación de la imagen. La ikonomía se refería, en Bizancio, al juego normal, adecuado, de las imágenes, de los íconos, conforme a reglas ideológicas que definían allí la imagen.

Peter Weibel, teórico y director del Centro de Arte y Tecnologías de la Información (ZKM), en la ciudad de Karlsruhe, rescata una primera formulación y delimitación conceptual que produce la interfaz:

En 1755 el físico matemático Roger Joseph Boscovich definió la hipótesis de la interfaz con mayor precisión: "nosotros no podemos reconocer un movimiento común que compartimos con el mundo [...] De hecho, incluso podría ocurrir que el mundo que yace en toda su extensión justo ante nuestros ojos se contrayera o expandiese en cuestión de días; pero, si esto realmente ocurriera, no se daría cambio alguno en la impresión sobre la mente y por lo tanto no habría ninguna percepción de tal cambio".

Es así que entonces tenemos en consideración los efectos y las variables de la construcción de una subjetividad que en un proyecto estatal desarrollista configura el enunciado de G.B. Ya mucho antes, en los inicios de esta configuración se produce una gran revolución social que en su interior traía la propuesta por una escuela activa, es la inédita convocatoria de la malograda asamblea nacional constituyente del marzo del año 1925, y su propuesta educativa que será implementada para todo el sistema durante los primeros diez meses del año 1928, con una violenta interrupción que también sacudirá lo que había alcanzado a lograr estructuralmente para las disciplinas proyectuales. Sin embargo a la caída de Carlos Ibáñez en el año 1931, esta ideología sindical funcionalista que asimilaba estructuras sociales a funcionamiento orgánico se sigue desarrollando como una opción opuesta al régimen liberal y al autoritarismo soviético. Entre estas resistencias al fenómeno de 1928, estaba el grupo de profesores agrupados en Curicó con el nombre de NERVIO. En el año 1936 publican el texto: El sindicalismo funcional en la teoría y en la práctica, donde hacen toda una relación de estos procesos. Un grupo de estos profesores serán designados a cargo de las Escuelas Consolidadas en los años cincuenta, base de donde saldrán las propuestas para la Escuela Nacional Unificada del año 1971.

Es en esta suma de variables de construcción de un modelo de sentido y realidad que el joven arquitecto Waldo Parraguez formula sus tesis epistemológicas publicadas en la revista ARQuitectura de 1935, respecto de la "plástica moderna", situada en un espacio que él deduce como "expresión plástica", derivada de la "proporción plástica", y que no sigue la deducción de esta hacia el "tema real", ni el "tema abstracto", que a su vez se suceden en "expresión tergiversada" e "incomprensión", respectivamente, para terminar en lo que para él es "literatura" y "vacío". 


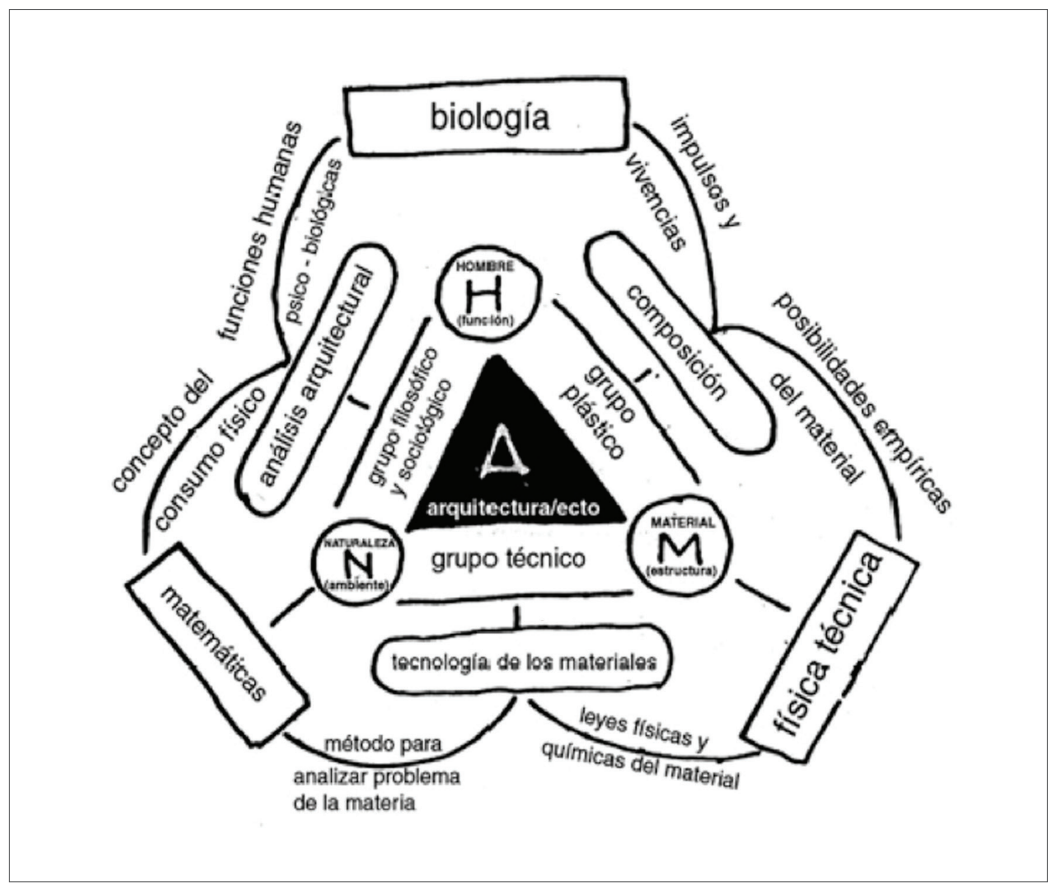

La susceptibilidad de construir esta otra noción de espacio independiente colaborativo, horizontal y descentralizado consigue después de 1945 una nueva gran concreción, con la reforma del arquitecto integral, que superaba anteriores implementaciones del modelo (modern style) por la construcción de un referente que incorporaba el trabajo en equipo, las referencias a Le Corbusier, Hannes Meyer y Walter Gropius, de una forma más extrema hacia una bioarquitectura, y hacer del espacio público una ciudad como "organismo vivo". La interfaz de la CORFO 1968-1973 no es sino posible por esta susceptibilidad construida desde lo que aproxima el enunciado del ingeniero MINVU Abraham Freifeld: una "ingeniería utópica”. Para un "neo constructivismo orgánico", desarrollado durante este período, en la constitución de este espacio de interacción como sistema social, y como construcción de realidad, o al menos su verosimilitud: la necesidad de articular una crítica a la economía política del espacio de lo simbólico como sistema de representación psicosocial institucional; heterárquico, descentrado, nodal.
Figura 11. Plan de estudios que retoma la idea de restitución del sujeto constructivo, concluyente y proyectual desde la figura del "Arquitecto Integral", a través de ciclos de análisis y síntesis, materializando los principios de la "Bioarquitectura".

Fuente: Schapira, González y Weiner (1947, p.43). 


\section{Bibliografía}

Aumont, J. (1992). El papel del dispositivo. En La Imagen. Barcelona: Paidós Comunicación.

Bonsiepe, G. (1972). VI/VI/SECCIÓN del diseño industrial. Revista INTEC, (2).

Bonsiepe, G. (1973). VI/ VI/ SECCIÓN del diseño industrial. $A \cup C A,(24 / 25)$.

Cardoso, A. (1972). Arte integrado. Revista de Educación MINEDUC, (39).

Drucker, P. (1993). La Sociedad Poscapitalista. Buenos Aires: Editorial Sudamericana.

Frente Funcional Sindicalista (1936). El sindicalismo funcional en la teoría y en la práctica. Santiago de Chile: Editorial Nervio.

Harnecker, M. et al. (1972). La participación de los trabajadores. Chile Hoy, (7-8).

Parraguez, W. (1935). Ensayo sobre Plástica Moderna. Revista arquitectura, (1).

Schapira, A., González, J. B. y Weiner, T. (1947). Facultad de Arquitectura, Universidad de Chile. Metodología y reglamentación del plan de estudios. Arquitectura y Construcción, (11).

Vargas-Koch, S. (2007). Anteproyecto centro comunal Valparaíso. Reflexiones sobre las relaciones de Bill, la HfG y América Latina. Santiago de Chile. Recuperado de http://www.magisterarq.cl/fileadmin/docs/ trayectoria_trabajos/Arquitecturas_america_latina/ anteproyecto_valparaiso_s.vargas.pdf (Marzo de 2015).

Weibel, P. (1999). El mundo como interfaz. La revolución digital y sus dilemas. El Paseante, (27-28), 110-120.

\section{Acrónimos y siglas}

BID: Banco Interamericano de Desarrollo

BM: Banco Mundial

CEPAL: Comisión Económica para América Latina y el Caribe Cybersyn o proyecto Synco: Sinergia Cybernética CORFO: Corporación de Fomento de la Producción (Chile) CORMU: Corporación de Mejoramiento Urbano CPM: Método de la Ruta Crítica ECOM: Empresa Nacional de Computación, filial CORFO G77: Grupo de los 77 países no pertenecientes a las grandes potencias de los bloques socialista y capitalista, durante la Guerra Fría

MINVU: Ministerio de la Vivienda y Urbanismo

ONU: Organización de Naciones Unidas

OІT: Organización Internacional del Trabajo

PERT: Software prospectivo

UNCTAD: United Nations Conference to Trade and

Development

UTE: Universidad Técnica del Estado

VSM: Modelo de Sistema Viable 